\title{
P16: Ernährung älterer Menschen in einer stationären Pflegeeinrichtung
}

\author{
Ingolf Adner · Jörg Klewer
}

Online publiziert: 30. Oktober 2013

(C) Springer-Verlag Wien 2013

Einleitung: Die Ernährungssituation pflegebedürftiger älterer Menschen in stationären Einrichtungen unterscheidet sich drei Jahre nach Implementierung des Expertenstandards „Ernährungsmanagement zur Sicherstellung und Förderung der oralen Ernährung in der Pflege" noch erheblich von der selbstständig lebender Seniorinnen und Senioren. Daher ist es geboten, die Ernährungssituation in Pflegeeinrichtungen wissenschaftlich zu analysieren, um daraus wirkungsvolle Maßnahmen zur Verbesserung abzuleiten.

Methodik: In einer Vollerhebung wurde die Umsetzung des Expertenstandards „Ernährungsmanagement zur Sicherstellung und Förderung der oralen Ernährung in der Pflege“ in einer stationären Pflegeeinrichtung evaluiert. Besondere Beachtung fanden dabei Pflegebedürftige mit einem Ernährungsrisiko bzw. manifester Mangelernährung.

Ergebnisse: Es wurde ersichtlich, dass zum Zeitpunkt der Untersuchung neben der in weiten Teilen erfolgreichen
Implementierung des Expertenstandards Defizite in der Struktur zu verzeichnen waren. Insbesondere bestand Entwicklungsbedarf hinsichtlich der individuellen Ernährungsproblematik sowie bei der Fortbildung der Mitarbeiterinnen und Mitarbeiter. Die Versorgung der Bewohnerinnen und Bewohner mit Nährstoffen nach den Kriterien der DGE war in einigen Punkten nicht gegeben.

Diskussion/Schlussfolgerung: Die individuellen Ernährungsgewohnheiten der Bewohnerinnen und Bewohner sind in der Pflegestruktur sowie in den Pflegeprozessen stärker zu berücksichtigen. Der Fortbildungsbedarf der Mitarbeiterinnen und Mitarbeiter zur Nahrungs- und Flüssigkeitsversorgung alter Menschen sollte durch die Einrichtungsleitung regelmäßig evaluiert und Fort- bzw. Weiterbildungen angeboten werden. Die Zufuhr von Makro- und Mikronährstoffen sowie die Flüssigkeitsaufnahme sollten sich vermehrt an den Empfehlungen der DGE orientieren.
I. Adner $(\triangle)$

Studiengang Pflegemanagement, Studienzentrum Dresden,

Hamburger Fern-Hochschule, Hamburg, Deutschland

E-Mail: ingolf.adner@campus.hamburger-fh.de

J. Klewer

Fakultät Gesundheits- und Pflegewissenschaften,

Westsächsische Hochschule Zwickau, Zwickau, Deutschland 